\title{
HATT: a phase IV, single-arm, open-label study of sorafenib in Taiwanese patients with advanced hepatocellular carcinoma
}

\author{
Shi-Ming Lin ${ }^{1} \cdot$ Sheng-Nan Lu ${ }^{2} \cdot$ Ping-Tsung $\mathrm{Chen}^{3} \cdot$ Long-Bin Jeng ${ }^{4} \cdot$ \\ Shinn-Cherng Chen ${ }^{5} \cdot$ Chi-Tan $\mathrm{Hu}^{6} \cdot$ Sien-Sing Yang ${ }^{7} \cdot$ Marie-Aude Le Berre ${ }^{8}$. \\ Xuan Liu' ${ }^{9}$ David Y. Mitchell ${ }^{10}$ - Klaas Prins ${ }^{11} \cdot$ Joachim Grevel ${ }^{12}$. \\ Carol A. E. Peña ${ }^{13} \cdot$ Gerold Meinhardt $^{14}$
}

Received: 14 March 2016/Accepted: 9 November 2016/Published online: 1 December 2016

(C) The Author(s) 2016. This article is published with open access at Springerlink.com

\begin{abstract}
Background Sorafenib significantly improves survival in patients with advanced hepatocellular carcinoma (HCC). This phase IV study assessed sorafenib efficacy/safety in Taiwanese patients with advanced HCC and Child-Pugh A status.

Methods All patients received $400 \mathrm{mg}$ sorafenib BID. Safety, efficacy, sorafenib pharmacokinetics, and ChildPugh progression were evaluated. A hand-foot skin reaction (HFSR) prevention substudy assessed HFSR
\end{abstract}

Electronic supplementary material The online version of this article (doi:10.1007/s12072-016-9774-x) contains supplementary material, which is available to authorized users. incidence and grade/severity and time to HFSR in 29 and 34 patients randomized to corticosteroid and noncorticosteroid ointments, respectively, and in 88 nonrandomized patients.

Results The 151 patients included $120(80 \%)$ male patients and $81(54 \%)$ with stage IV disease. Mean sorafenib dose was $626 \mathrm{mg} / \mathrm{day}$, and median treatment duration was 4.2 months. Median overall survival (OS), progression-free survival, and time to progression (TTP) were $8.6,2.7$, and 3.8 months, respectively. Disease control and response rates (partial responses only) were 48 and $6.6 \%$, respectively. Median TTP from Child-Pugh A to B/C was 88 days. Drug-related adverse events (AEs) occurred in $89.4 \%$ of patients; none were new or unexpected. The most

Klaas Prins

klaas.prins@qppharmetra.com

Joachim Grevel

jgrevel@btconnect.com

Carol A. E. Peña

carol.pena@bayer.com

Gerold Meinhardt

gerold.meinhardt@bayer.com

1 Division of Hepatology, Department of Gastroenterology and Hepatology, Linkou and Taipei Chang Gung Memorial Hospital Chang Gung University, No. 5 Fuhsing Street, Kuei Shan Hsiang, Taoyuan, Taiwan

2 Division of Hepato-Gastroenterology, Kaohsiung Chang Gung Memorial Hospital, 100 Tapei Road, Niaosung, Kaohsiung 833, Taiwan

3 The Division of Hematology \& Oncology, Chiayi Chang Gung Memorial Hospital, 6 W Sec, Chia Pu Road, $\mathrm{Pu}-\mathrm{Tz}$ City 613, Chiayi, Taiwan

4 Organ Transplantation Center, China Medical University Hospital, North District, Taichung City 404, Taiwan 
frequent grade $\geq 3$ drug-related, treatment-emergent AEs were HFSR (13.2\%), diarrhea (11.9\%), and hypertension $(6.6 \%)$. Corticosteroid ointment tended to reduce the severity and incidence of all HFSR-associated parameters. Pharmacokinetic exposure was unaltered by Child-Pugh progression. The final pharmacokinetic model predicted 13.1 and $33.8 \%$ reductions in sorafenib exposure over 6 and 12 months, respectively.

Conclusions There was a trend of longer OS and TTP in Taiwanese patients with advanced HCC compared with patients with advanced HCC in the Asia-Pacific trial. Sorafenib exposure did not correlate with liver function. Reduced pharmacokinetic exposure over time was unrelated to reduced or interrupted dosing.

Keywords Sorafenib - Advanced hepatocellular carcinoma - Metastatic hepatocellular carcinoma · Taiwanese patients - Overall survival - Time to progression

\section{Introduction}

Hepatocellular carcinoma (HCC) is the sixth most common cancer worldwide and the third most common in the AsiaPacific region [1, 2]. Geographical differences in $\mathrm{HCC}$ incidence are largely due to variations in hepatitis $\mathrm{B}$ and $\mathrm{C}$ infection [3, 4]. In East Asia, including Taiwan, where

5 Division of Hepato-Biliary-Pancreatic Medicine, Kaohsiung Medical University Hospital, No. 100, Tzyou 1st Road, Kaohsiung 807, Taiwan

6 Division of Hepato-Gastroenterology, Buddhist Tzu Chi General Hospital, Tzu Chi University, No. 707, Sec. 3 Chung Yang Road, Hualian City 970, Hualien County, Taiwan

7 Liver Center, Cathay General Hospital, Taipei and Medical Faculty, Fu-Jen Catholic University, No. 280, Sec. 4 Jen-Ai Road, Taipei 10630, Taiwan

8 Clinical Statistics, Bayer HealthCare Pharmaceuticals, Medical Affairs, Building C2 1.11, 220 Ave de la Recherche, Loos 59120, France

9 Global Clinical Development, Bayer HealthCare Co. Ltd., 18F Tower B, Bayer Center, No. 27, Dong San Huan North Road, Chaoyang District, Beijing, China

10 Mitchell Pharmaceutical Consulting, 1188 Hawk Ridge Road, Lafayette, CO 80026, USA

11 qPharmetra, LLC, Kwakkenbergweg 39, 6523, MK, Nijmegen, The Netherlands

12 BAST Inc Limited, 7 Wellingtonia Close, Shepshed LE12 9FB, UK

13 Clinical Sciences, Bayer Healthcare Pharmaceuticals, 100 Bayer Road, Whippany, NJ, USA

14 Global Clinical Development Oncology, Bayer Healthcare Pharmaceuticals, 100 Bayer Road, Whippany, NJ, USA hepatitis $\mathrm{B}$ virus (HBV) is endemic, the incidence rate of HCC is $20-28$ per 100,000 people. Approximately $10-20 \%$ of cases of $\mathrm{HCC}$ occur in patients with chronic $\mathrm{HBV}$ infection in the absence of cirrhosis.

Two randomized, placebo-controlled, phase III trials, the Sorafenib HCC Assessment Randomized Protocol (SHARP) and the Sorafenib Asia-Pacific (Sorafenib AP) trials, showed that the multikinase inhibitor sorafenib significantly improves overall survival (OS) and progressionfree survival (PFS) in patients with advanced HCC [5, 6]. Based on these results, sorafenib was approved as systemic treatment for patients with advanced HCC and remains the only globally approved systemic treatment for this disease.

As a post-approval commitment in Taiwan, 151 patients were enrolled in this phase IV, single-arm study [Hepatocellular carcinoma-Advanced stage-sorafenib Trial in Taiwanese patients (HATT)] to confirm the efficacy and safety of sorafenib. The requested number of patients was based on the number of patients from the mainland of China who were treated in the phase 3 Asia-Pacific study (NCT00492752), which was 151 of the 226 randomized patients [5]. The main objective of the post-authorization study was to evaluate the safety and efficacy profile of sorafenib and to evaluate Child-Pugh status progression in Taiwanese patients with advanced HCC treated with sorafenib. The main study did not have a primary endpoint. Another objective of this main study was to assess the pharmacokinetics of sorafenib in patients with HCC. A substudy of hand-foot skin reaction (HFSR) prevention assessed HFSR incidence and grade/severity and time to HFSR in patients randomized to corticosteroid and noncorticosteroid ointment and in a group of nonrandomized, untreated patients.

\section{Methods}

This prospective, open-label, single-arm, post-authorization study was conducted across seven sites in Taiwan in patients with advanced HCC. All patients meeting entry criteria received sorafenib $400 \mathrm{mg}(2 \times 200-\mathrm{mg}$ tablets $)$ twice daily (BID) on a continuous schedule. For the purpose of data recording, treatment period was divided into 21-day cycles. Treatment continued beyond radiologic progression, provided the subject derived clinical benefit, as judged by the treating physician.

\section{Ethical considerations}

The trial protocol was approved by the institutional review boards of all seven institutions. All patients provided written informed consent. The trial has been registered at clinicaltrials.gov as NCT01098760. 


\section{Inclusion criteria}

Patients aged $\geq 18$ years were included if they had cytologically or histologically documented, unresectable advanced and/or metastatic HCC not amenable to local treatment methods, with at least one tumor lesion that could be measured accurately in at least one dimension and had not been treated with local therapy. HCC in cirrhotic patients was diagnosed by American Association for the Study of Liver Disease criteria; noncirrhotic patients required histological or cytologic confirmation. Patients who previously received local therapy (e.g., surgery, radiation, hepatic artery embolization, transarterial chemoembolization, radiofrequency ablation, percutaneous ethanol injection, or cryoablation) were deemed eligible, but local therapy had to be completed at least 4 weeks before study entry, and all toxic effects of any prior local treatments had to have resolved. Moreover, previously treated lesions could not be selected as target lesions. Other inclusion criteria included Child-Pugh Class A, Eastern Cooperative Oncology Group performance status of $0-2$, and life expectancy $\geq 12$ weeks. The first patient's first visit was in August 2010 and the last patient's first visit was in June 2012.

\section{Exclusion criteria}

Patients were excluded if they had previous or concurrent cancer, unless curatively treated $>3$ years before study entry; renal failure requiring dialysis; a history of cardiac disease, including congestive heart failure, active coronary artery disease, or cardiac arrhythmias; or uncontrolled hypertension (systolic blood pressure $>150 \mathrm{mmHg}$ or diastolic blood pressure $>90 \mathrm{mmHg}$ ). Also excluded were patients with active, clinically serious infections [except for HBV/hepatitis C virus (HCV)]; central nervous system tumors; clinically significant gastrointestinal bleeding $<30$ days before study entry; history of organ allograft transplantation; Child-Pugh Class B or C liver status; or uncontrolled ascites. Patients previously treated with yttrium-90 spheres, those with clinically significant peripheral vascular disease or a history of substance abuse or psychological conditions interfering with participation, and patients unable to take oral medications were also excluded.

\section{Dose modifications}

All patients were initially treated with $400 \mathrm{mg}$ sorafenib BID. Reductions to $400 \mathrm{mg}$ once daily and $400 \mathrm{mg}$ every other day (QOD) were permitted for clinically significant hematologic and nonhematologic toxicities. Treatment was decreased one dose level for grade 3 hematologic toxicities. Treatment was interrupted in patients with grade 4 hematologic toxicities and in patients with grade 3 nonhematologic toxicities (except skin toxicity and hypertension) until they achieved grade $\leq 2$ with treatment resumed at one lower dose level. Treatment was also discontinued in patients with grade 4 nonhematologic toxicities and in patients who would have required further dose reductions beyond $400 \mathrm{mg}$ QOD. In patients who experienced two or more toxicities, reductions were according to the toxicity with the highest grade; alternatively, if both toxicities were of equal grade, dose was reduced according to the toxicity deemed most causally related to study treatment. Increases were permitted in patients who previously had dose reductions and remained on stable doses for $\geq 3$ weeks without further toxicities, although only one such increase per patient was permitted.

\section{Safety and efficacy assessments}

Safety was evaluated at screening (within 28 days of first dose of study drug), before dosing on the first day of study drug administration, and before dosing every 3 weeks ( \pm 7 days). Tumors were assessed within 28 days of the start of study drug using CT or MRI scans. For the purpose of data recording, the treatment period was divided into 3 -week cycles. Tumor measurements and evaluation of tumor response were performed within the last 7 days of every other treatment cycle, beginning with the second cycle. Child-Pugh status was determined at screening and on day 1 of each cycle.

\section{Follow-up}

After completion of study drug treatment, patients were contacted every 3 months to determine survival status. Follow-up was continued until 6 months after the first treatment of the last patient. For regulatory purposes, the end of the trial was defined as 6 months after the first treatment of the last patient but not before 106 patients had died.

\section{Pharmacokinetic analysis}

After enrollment started, the study protocol was amended to include a steady-state pharmacokinetic analysis, as requested by the Taiwan Department of Health. Sorafenib pharmacokinetics were assessed in a subpopulation of 85 patients. Blood samples were collected on day 1 of each 21-day cycle, starting with cycle 2 , to assess sorafenib exposure concurrently with each determination of ChildPugh status. Sorafenib concentrations in plasma were determined by high-pressure liquid chromatography and tandem mass spectrometry. Summary statistics were 
generated describing sorafenib plasma concentrations of all pharmacokinetics samples at a given Child-Pugh score.

Plasma sorafenib concentrations were analyzed using a previously defined population pharmacokinetic model $[7,8]$ and the software program, NONMEM v.7.2 (ICON, Dublin, Ireland). Briefly, the previously developed model applied to the HATT data used a 1-compartment model with three sequential transit absorption compartments and included sex, body weight, body mass index, race (Asian/ nonAsian), Child-Pugh score, age, liver transaminases, albumin, bilirubin, alkaline phosphatase, lactate dehydrogenase, serum glutamic oxaloacetic transaminase, serum glutamic pyruvic transaminase, prothrombin time, creatinine clearance, co-medication with CYP3A4 or UGT1A9 inducers or inhibitors, or thyroxine as a covariate for sorafenib apparent clearance (CL/F). For the current analyses, gender was the only covariate included in the model because it was the one covariate that significantly influenced $\mathrm{CL} / \mathrm{F}$ in the meta-analysis that was performed to develop the population pharmacokinetic model. Predicted sorafenib concentrations were calculated based on daily doses and dose reductions, interruptions, and discontinuations. Observed and predicted sorafenib concentrations from the final model were compared, as were changes over time.

\section{Incidence of and time to hand-foot skin reaction}

During the HATT trial, the study protocol was amended to include assessment of the impact of two different ointments on HFSR prevention. Beginning at the start of treatment, 29 patients were randomized to a corticosteroid and 34 to a noncorticosteroid ointment, to be applied twice daily to soles and palms for 21 days. The rates of HFSR after 3 and 6 weeks and overall, as well as time to HFSR and grade/severity of HFSR, were compared in these two groups and with a group of 88 patients enrolled before the start of the HFSR prevention substudy. Exposure to sorafenib was also assessed in these three subgroups, as measured by duration of treatment, total dose, and number of dose modifications.

\section{Statistical analysis}

Demographic variables and baseline characteristics were summarized descriptively for all patients in the intent-totreat population. Continuous variables were reported as mean \pm standard deviation or medium (minimum, maximum), and categorical variables were reported as frequency (\%). Time to event variables, including OS, PFS, time to progression (TTP), and time from Child-Pugh A to Child-Pugh B/C, were assessed by the Kaplan-Meier method and compared by the log-rank test. Treatment- emergent adverse events (AEs), drug-related AEs, AEs leading to premature termination, dose reductions and interruptions, serious AEs (SAEs), and laboratory parameters were summarized using descriptive statistics for safety.

\section{Results}

\section{Patient characteristics and exposure to sorafenib}

Most patients (54\%) had stage IV disease at study entry (Table 1). The primary causes of HCC in these patients were HBV infection $(n=81,53.6 \%)$ and $\mathrm{HCV}$ infection ( $n=41,27.2 \%$; Table 1$)$. Of the 151 patients, $23(15.3 \%)$ were continuing treatment at the time of database cutoff, whereas $128(84.8 \%)$ terminated treatment, most for AEs, disease progression/recurrence, and withdrawal of consent (Fig. 1).

Although the study protocol required that all patients be Child-Pugh class A, two (1.3\%) were Child-Pugh class B at baseline. Protocol deviations were documented for both patients.

Assessment of time of patient exposure to sorafenib showed that the median duration of treatment was 18.1 weeks (range, 0.3-124 weeks), and the mean \pm SD duration of treatment was $29.9 \pm 30.3$ weeks. During this time, patients received a median $661.5 \mathrm{mg} /$ day (range, 211.5-800 mg/day) and a mean $\pm \mathrm{SD}$ of $625.9 \pm 170.3 \mathrm{mg} /$ day sorafenib. Of the 151 patients, 112 $(74.2 \%)$ required dose reductions, and $99(65.8 \%)$ required dose interruptions.

\section{Efficacy}

Median OS was 8.6 months (95\% CI, 6.4-10.1 months); median PFS was 2.7 months (95\% CI, 2.6-3.9 months); and median TTP was 3.8 months $(95 \%$ CI, 2.6-4.1 months; Fig. 2).

Ten patients $(6.6 \%)$ showed a partial response to treatment, and $62(41.1 \%)$ had confirmed stable disease. Thus, the overall response rate was $6.6 \%$ and the disease control rate was $47.7 \%$. Descriptive analysis of mean alpha fetoprotein (AFP) levels showed that AFP levels were variable but decreased from baseline by treatment cycle 6 (Supplemental Fig. 1). At the end of treatment, 73 patients were classified as Child-Pugh A, 50 as Child-Pugh B, and 12 as Child-Pugh C. The median time to worsening of liver function, from Child-Pugh A to first detection of ChildPugh B or C, was 2.9 months (95\% CI, 1.9-4.8 months) (Fig. 2). Child-Pugh scores of individual patients did not consistently progress over time, from $\mathrm{A}$ to $\mathrm{B}, \mathrm{B}$ to $\mathrm{C}$, and $\mathrm{A}$ to $\mathrm{C}$; rather, Child-Pugh scores varied within a patient 
Table 1 Demographic characteristics and disease history of Taiwanese patients with HCC

\begin{tabular}{|c|c|}
\hline Characteristics & Total patients $(n=151)$ \\
\hline Median age, year (range) & $62.0(28-97)$ \\
\hline Male patients, $n(\%)$ & $120(79.5)$ \\
\hline Mean \pm SD weight, $\mathrm{kg}$ & $62.35 \pm 10.55$ \\
\hline Mean \pm SD BMI, $\mathrm{kg} / \mathrm{m}^{2}$ & $23.58 \pm 3.26$ \\
\hline \multicolumn{2}{|l|}{ Etiology, $n(\%)$} \\
\hline Hepatitis B & $81(53.6)$ \\
\hline Hepatitis C & $41(27.2)$ \\
\hline Alcohol use & $6(4.0)$ \\
\hline Hepatitis B and C & $5(3.3)$ \\
\hline Hepatitis B and alcohol & $3(2.0)$ \\
\hline Nonalcoholic steatohepatitis & $2(1.3)$ \\
\hline Hepatitis $\mathrm{C}$ and alcohol & $1(0.7)$ \\
\hline Hepatitis B and C and alcohol & $1(0.7)$ \\
\hline Unknown & $11(7.3)$ \\
\hline \multicolumn{2}{|l|}{ ECOG PS, $n(\%)$} \\
\hline 0 & $123(81.5)$ \\
\hline 1 & $27(17.9)$ \\
\hline 2 & $1(0.7)$ \\
\hline \multicolumn{2}{|l|}{ Child-Pugh classification, $n(\%)$} \\
\hline A & $149(98.7)$ \\
\hline B & $2(1.3)$ \\
\hline \multicolumn{2}{|l|}{ Child-Pugh Score, $n(\%)$} \\
\hline 5 & $80(53.0)$ \\
\hline 6 & $69(45.7)$ \\
\hline 7 & $1(0.7)$ \\
\hline 8 & $1(0.7)$ \\
\hline \multicolumn{2}{|l|}{ Disease stage, $n(\%)$} \\
\hline II & $8(5.3)$ \\
\hline IIIA & $32(21.2)$ \\
\hline IIIB & $17(11.3)$ \\
\hline IIIC & $13(8.6)$ \\
\hline IV & $81(53.6)$ \\
\hline Liver cirrhosis & $144(95.4)$ \\
\hline AFP > $200 \mathrm{ng} / \mathrm{mL}$ & 99 (65.6) \\
\hline
\end{tabular}

$A F P$ alpha-fetoprotein, BMI body mass index, ECOG PS Eastern Cooperative Oncology Group performance score, $H C C$ hepatocellular carcinoma, $S D$ standard deviation

between visits, with some patients showing improvements after initial worsening and others showing progressive deterioration.

\section{Safety}

There were no unexpected AEs. Drug-related treatmentemergent AEs were reported in 135 patients (89.4\%), with grade 1/2 drug-related AEs occurring in 63 patients (41.7\%) and grade 3/4 drug-related AEs occurring in 72

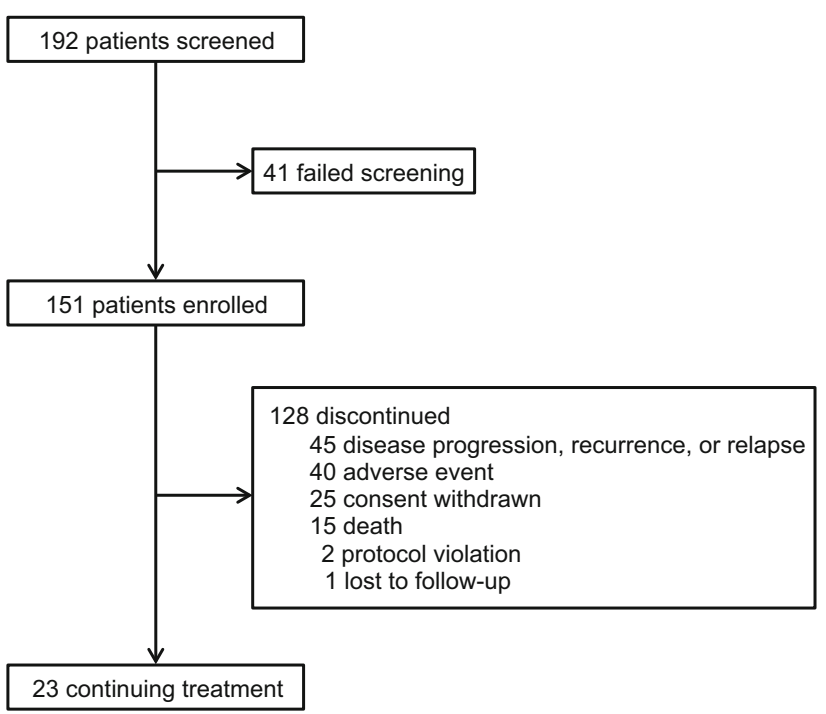

Fig. 1 CONSORT diagram

patients $(47.7 \%)$ (Table 2). The most frequently reported drug-related, treatment-emergent AEs in this trial were HFSR (64.9\%), diarrhea (45.0\%), and ascites (27.2\%), whereas the most frequently reported grade 3 drug-related, treatment-emergent AEs were HFSR (13.2\%), diarrhea $(11.9 \%)$, and hypertension (6.6\%). Treatment-emergent SAEs were reported in 110 patients $(72.8 \%)$, and drugrelated treatment-emergent SAEs in $14(9.3 \%)$. Grades 3 and 4 drug-related, treatment-emergent SAEs were reported in $10(6.6 \%)$ and $3(2.0 \%)$ patients, respectively, but there were no drug-related, treatment-emergent grade 5 SAEs.

\section{Pharmacokinetics}

Of the 151 patients, $85(56.3 \%)$ provided a total of 847 plasma samples for pharmacokinetic analysis. Median sorafenib concentrations at the time of pharmacokinetic sampling of patients classified as Child-Pugh A, B, and C were $3.72 \mathrm{mg} / \mathrm{L}$ (range, $0.0162-15.6 \mathrm{mg} / \mathrm{L}$ ), $2.90 \mathrm{mg} / \mathrm{L}$ (range, $0.0124-15.6 \mathrm{mg} / \mathrm{L}$ ), and $3.29 \mathrm{mg} / \mathrm{L}$ (range, 0.0287-7.44 mg/L), respectively (Supplemental Fig. 2). Application to the HATT trial of a population pharmacokinetic model developed from 10 phase I to III trials of sorafenib in healthy volunteers and in patients with renal cell carcinoma, HCC, differentiated thyroid cancer, and other tumor types, including patient sex as a covariate with sorafenib CL/F [8], suggested a trend toward decreasing sorafenib concentrations over time that was not accounted for by the model (Supplemental Fig. 3). Examination of sex as a covariate indicated a $40.5 \%$ lower overall CL/F in female than in male patients. Linear and power relationships for a change in clearance or bioavailability over time were tested, with the power model providing the best fit. 
(a)

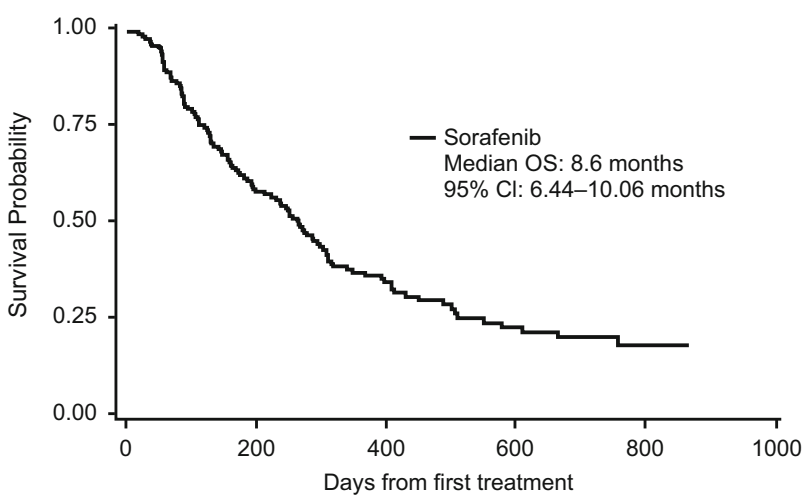

(c)

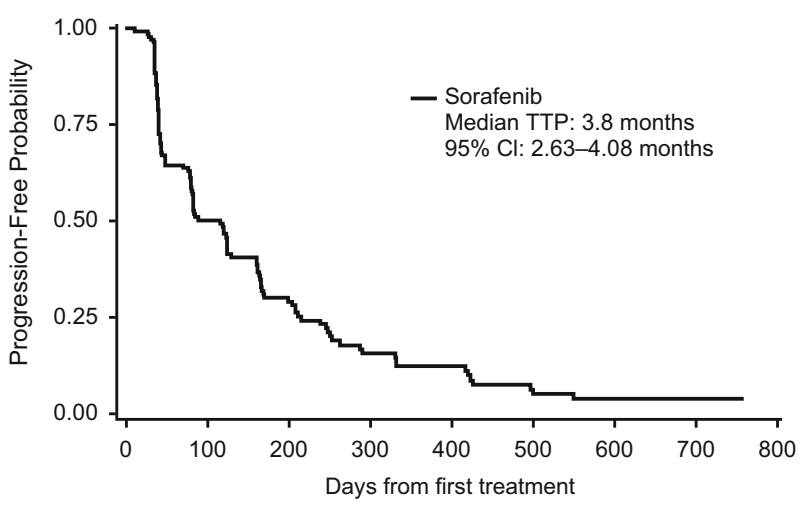

Fig. 2 Efficacy outcomes. Kaplan-Meier plots of a OS, b PFS, c TTP, and d time from Child-Pugh A to Child-Pugh B/C liver status in patients enrolled in the HATT trial. $O S$ overall survival, PFS

Table 2 Percentage of patients with drug-related, treatment-emergent AEs observed in $>5 \%$ of patients

\begin{tabular}{lll}
\hline AE & Total & \%Grade 3/4 \\
\hline HFSR & 64.9 & $13.2 / 0$ \\
Diarrhea & 45.0 & $11.9 / 0$ \\
Alopecia & 27.2 & $0 / 0$ \\
Hypertension & 18.5 & $6.6 / 0$ \\
Fatigue & 11.3 & $0.7 / 0$ \\
Rash maculopapular & 11.3 & $0.7 / 0$ \\
Platelet count decreased & 9.9 & $4.0 / 1.3$ \\
Pruritus & 8.6 & $0.7 / 0$ \\
AST increased & 6.6 & $4.0 / 1.3$ \\
Anorexia & 6.0 & $0 / 0$ \\
Hoarseness & 5.3 & $0 / 0$ \\
Weight loss & 5.3 & $0 / 0$ \\
\hline
\end{tabular}

AEs adverse events, $A S T$ aspartate aminotransferase, HFSR hand-foot skin reaction

Inclusion of albumin and bilirubin as covariates improved the model fit, whereas alkaline phosphatase, prothrombin time, international normalized ratio, serum glutamic (b)

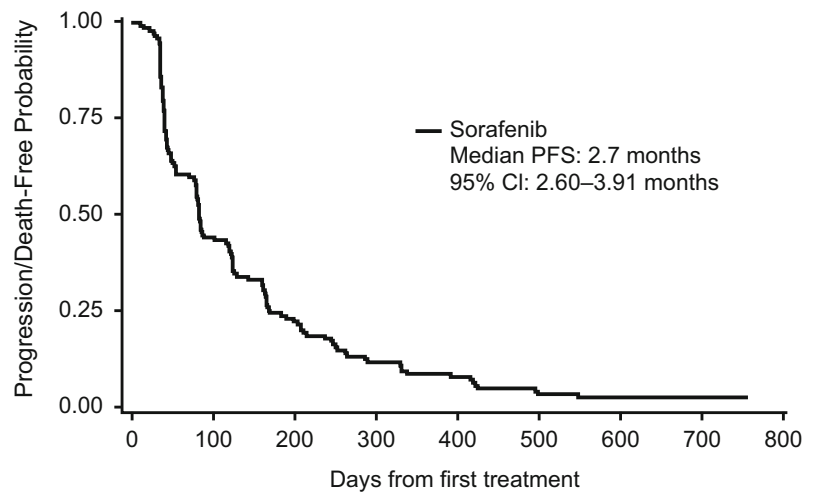

(d)

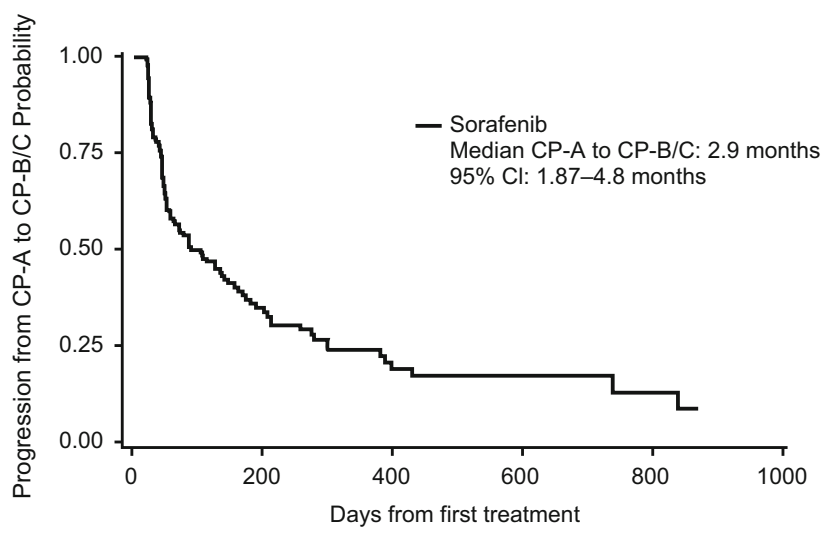

progression-free survival, TTP time to progression, HATT Hepatocellular Carcinoma-Advanced Stage-Sorafenib Trial in Taiwanese patients

oxaloacetic transaminase, serum glutamic pyruvic transaminase, and Child-Pugh score did not affect sorafenib exposure. Both bilirubin and albumin concentrations were positively correlated with sorafenib concentrations, although only bilirubin showed a time dependence, with sorafenib concentrations and bilirubin levels decreasing together over time. Using the final population pharmacokinetic model, the observed and predicted plasma sorafenib concentrations in individual patients demonstrated concordance over the entire time range studied (Table 3, Fig. 3). The final pharmacokinetic model predicted reductions of $13.1 \%$ and $33.8 \%$ in sorafenib exposure over 6 and 12 months, respectively.

\section{Hand-foot skin reaction substudy}

The incidence of overall HFSR and at weeks 3 and 6 did not differ significantly between patients randomized to corticosteroid and noncorticosteroid ointments (Table 4). HFSR incidence rates were also not significantly different between patients randomized to corticosteroid cream and those not treated with ointment (Table 4). HFSR scores at 3 
Table 3 Observed and predicted sorafenib exposure in patients over time

\begin{tabular}{llrrlll}
\hline Type & Time (months) & $n_{\text {obs }}$ & $n_{\text {ind }}$ & Geometric mean $(\mathrm{mg} / \mathrm{L})$ & $95 \%$ CI & Diff $(\%)$ \\
\hline Observed & $0-3$ & 332 & 85 & 3.42 & $3.07-3.81$ & 8.42 \\
& $3-6$ & 188 & 55 & 3.15 & $2.83-3.51$ & 0 \\
& $6-9$ & 125 & 35 & 2.81 & $2.40-3.33$ & -10.7 \\
& $9-12$ & 95 & 25 & 2.47 & $2.11-2.88$ & -21.7 \\
& $12-15$ & 56 & 18 & 2.23 & $1.80-2.76$ & -29.4 \\
& $15-18$ & 31 & 9 & 1.84 & $1.34-2.52$ & -41.7 \\
& $18-21$ & 20 & 6 & 2.02 & $1.40-2.91$ & -36.0 \\
Predicted & $0-3$ & 332 & 85 & 3.46 & $3.14-3.81$ & 14.7 \\
& $3-6$ & 188 & 55 & 3.01 & $2.76-3.30$ & 0 \\
& $6-9$ & 125 & 35 & 2.72 & $2.41-3.08$ & -9.62 \\
& $9-12$ & 95 & 25 & 2.62 & $2.33-2.94$ & -13.1 \\
& $12-15$ & 56 & 18 & 2.26 & $1.97-2.60$ & -25.0 \\
& $15-18$ & 31 & 9 & 2.00 & $1.61-2.48$ & -33.8 \\
& $18-21$ & 20 & 6 & 1.79 & $1.34-2.40$ & -40.6 \\
\hline
\end{tabular}

$\operatorname{Diff}(\%)$ the percentage difference in mean sorafenib exposure relative to the mean sorafenib exposure in the 3- to 6-month period

$C I$ confidence interval, $n_{\text {obs }}$ number of observations, $n_{\text {ind }}$ number of individuals

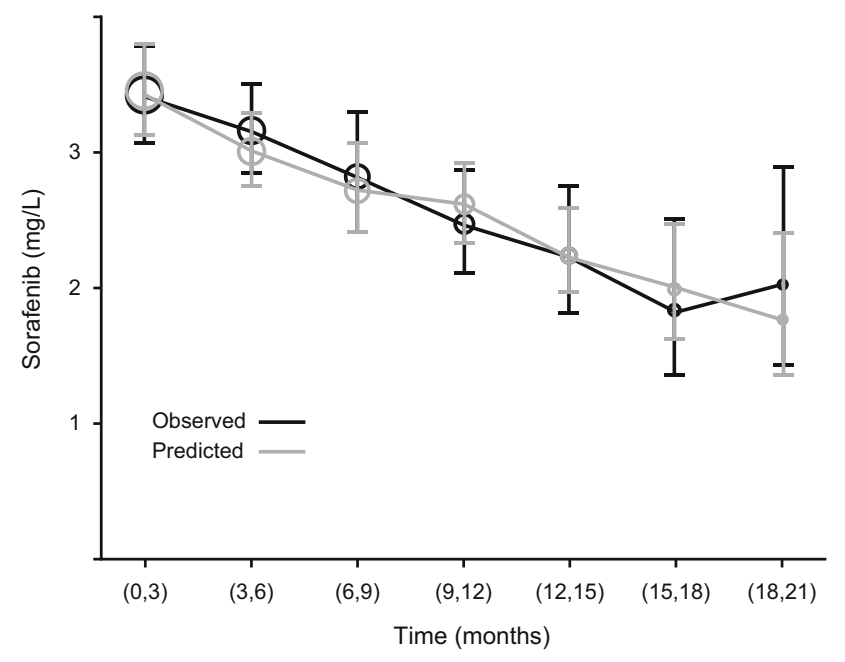

Fig. 3 Observed and predicted sorafenib concentrations over time. The final pharmacokinetics model predicted reductions in sorafenib exposure of 13.1 and $33.8 \%$ over 6 and 12 months of treatment, respectively, as shown by comparing the 3- to 6-month interval with the 9- to 12- and 15- to 18-month intervals. Time was stratified by quarter, with predicted concentrations representing individual values. Data are presented as geometric means and $95 \%$ confidence intervals

and 6 weeks did not differ significantly between patients randomized to corticosteroid and noncorticosteroid treatment, and between patients randomized to corticosteroid treatment and those not treated with ointment (Table 4). However, overall HFSR scores were significantly different between patients randomized to corticosteroid cream and noncorticosteroid ointments ( 0.83 vs. $1.26, p=0.031)$ and between patients randomized to corticosteroid cream and those not treated with ointment ( 0.83 vs. $1.24, p=0.038)$
(Table 4). Time to HFSR was longer in the group treated with corticosteroid cream (41 days) than in the group treated with noncorticosteroid cream (22 days) and the untreated control group (21 days), although these differences were not statistically significant. The incidence of grade 3 HFSR was low in both arms of the substudy (5.9\% in the noncorticosteroid group and $0 \%$ in the corticosteroid group; no grade 4 or 5 events were reported). However, the incidence of grade 3 HFSR (20.5\%) was higher in the nonointment group (Table 4). Corticosteroid treatment tended to reduce the severity and incidence of all HFSRassociated parameters (Fig. 4).

\section{Discussion}

As a post-approval commitment, 151 patients from Taiwan were enrolled in this phase IV, single-arm study to confirm the efficacy and safety of $400 \mathrm{mg}$ BID sorafenib in patients with advanced HCC, including patients with metastatic disease, those deemed unresectable and ineligible for local treatment, and those with local treatment failure. The results for OS, PFS, and TTP mirrored or exceeded those observed in the Sorafenib AP trial. For example, patients in this trial had a median OS of 8.6 months $(95 \% \mathrm{CI}$, 6.44-10.06 months) and a median TTP of 3.8 months (95\% CI, 2.63-4.08 months). In comparison, sorafenibtreated patients in the AP trial had a median OS of 6.5 months (95\% CI, 5.56-7.56 months) and a median TTP of 2.8 months (95\% CI, 2.63-3.58 months) [5]. However, median OS and TTP in this trial were in line with the sorafenib control arms of Asian patients in recent phase III 
Table 4 Incidence of grade 3-5 treatment-emergent AEs reported for $>10 \%$ of patients in any study arm and incidence of HFSR and HFSR scores in patients treated with corticosteroid and noncorticosteroid ointments and in nonointment-treated patients from the HATT trial registered prior to the beginning of the HFSR prevention substudy

\begin{tabular}{|c|c|c|c|c|c|c|}
\hline & \multicolumn{4}{|c|}{ Randomized into substudy } & \multicolumn{2}{|c|}{ Nonrandomized } \\
\hline & \multicolumn{2}{|c|}{$\begin{array}{l}\text { Corticosteroid group } \\
(n=29)\end{array}$} & \multicolumn{2}{|c|}{$\begin{array}{l}\text { Non-corticosteroid group } \\
(n=34)\end{array}$} & \multicolumn{2}{|c|}{$\begin{array}{l}\text { Nonointment group } \\
(n=88)\end{array}$} \\
\hline \multicolumn{7}{|l|}{ TEAE, $n(\%)$} \\
\hline Anemia & \multicolumn{2}{|c|}{$2(6.9)$} & \multicolumn{2}{|l|}{$4(11.8)$} & \multicolumn{2}{|l|}{$11(12.5)$} \\
\hline Abdominal pain & \multicolumn{2}{|c|}{$1(3.4)$} & \multicolumn{2}{|l|}{$3(8.8)$} & \multicolumn{2}{|l|}{$13(14.8)$} \\
\hline Ascites & \multicolumn{2}{|c|}{$3(10.3)$} & \multicolumn{2}{|l|}{$2(6.1)$} & \multicolumn{2}{|l|}{$13(14.8)$} \\
\hline Diarrhea $^{a}$ & \multicolumn{2}{|c|}{$3(10.3)$} & \multicolumn{2}{|l|}{$5(14.7)$} & \multicolumn{2}{|l|}{$11(12.5)$} \\
\hline Hepatic failure & \multicolumn{2}{|c|}{$1(3.4)$} & \multicolumn{2}{|l|}{$1(2.9)$} & \multicolumn{2}{|l|}{$12(13.6)$} \\
\hline Elevated alanine aminotransferase & \multicolumn{2}{|c|}{$3(10.3)$} & \multicolumn{2}{|l|}{$2(5.9)$} & \multicolumn{2}{|l|}{$12(13.6)$} \\
\hline $\begin{array}{l}\text { Elevated aspartate } \\
\text { aminotransferase }\end{array}$ & \multicolumn{2}{|c|}{$4(13.8)$} & \multicolumn{2}{|l|}{$5(14.7)$} & \multicolumn{2}{|l|}{$24(27.3)$} \\
\hline Elevated blood bilirubin & \multicolumn{2}{|c|}{$3(10.3)$} & \multicolumn{2}{|l|}{$4(11.8)$} & \multicolumn{2}{|l|}{$19(21.6)$} \\
\hline Decreased platelet count & 2( & & $4(11.8)$ & & $11(12.5)$ & \\
\hline Hyponatremia & 2( & & $7(20.6)$ & & $12(13.6)$ & \\
\hline Hypophosphatemia $^{a}$ & 3( & & $1(2.9)$ & & $4(4.5)$ & \\
\hline Encephalopathy & 1( & & $4(11.8)$ & & $12(13.6)$ & \\
\hline $\mathrm{HFSR}^{\mathrm{a}}$ & 0 & & $2(5.9)$ & & $18(20.5)$ & \\
\hline Hypertension $^{\mathrm{a}}$ & 5( & & $5(14.7)$ & & $9(10.2)$ & \\
\hline & & & & $p$ value $^{\mathrm{b}}$ & & $p$ value $^{\mathrm{c}}$ \\
\hline HFSR, $n(\%)$ & & & & & & \\
\hline 3 weeks & & $10(34.5)$ & $16(47.1)$ & 0.1561 & $44(50.0)$ & 0.0730 \\
\hline 6 weeks & & $15(51.7)$ & $20(58.8)$ & 0.2860 & $52(59.1)$ & 0.2434 \\
\hline Overall & & $16(55.2)$ & $24(70.6)$ & 0.1026 & $58(65.9)$ & 0.1492 \\
\hline Grade $3 \mathrm{HFSR}^{\mathrm{a}}, n(\%)$ & & & & & & \\
\hline 3 weeks & & $0(0)$ & $1(2.9)$ & N.D. & $11(12.5)$ & N.D. \\
\hline 6 weeks & & $0(0)$ & $2(5.9)$ & N.D. & $13(14.8)$ & N.D. \\
\hline Overall & & $0(0)$ & $2(5.9)$ & N.D. & $18(20.5)$ & N.D. \\
\hline HFSR score & & & & & & \\
\hline 3 weeks & & 0.55 & 0.74 & 0.2026 & 0.86 & 0.0744 \\
\hline 6 weeks & & 0.72 & 1.00 & 0.1159 & 1.03 & 0.0784 \\
\hline Overall & & 0.83 & 1.26 & 0.0314 & 1.24 & 0.0380 \\
\hline Median time to HFSR onset, $d$ (ran & & $41(1-238)$ & $22(5-145)$ & 0.0639 & 21 & 0.0782 \\
\hline
\end{tabular}

All $p$ values are 1-sided

HFSR hand-foot skin reaction, N.D. not determined, TEAE treatment-emergent adverse events

${ }^{a}$ No grade 4 or 5 AEs were reported

b Comparisons of patients randomized to corticosteroid cream and noncorticosteroid ointments

${ }^{c}$ Comparisons of patients randomized to corticosteroid cream and those not treated with ointment

trials investigating sunitinib and brivanib in advanced HCC $[9,10]$. The trend toward longer survival of patients with advanced HCC enrolled in current compared with earlier clinical trials may be due to better disease characteristics at enrollment and more physician experience handling the AEs associated with sorafenib.

Patients with advanced HCC are prone to deterioration of liver function, largely due to worsening of underlying cirrhosis and/or progression of intrahepatic tumor lesions, but in some patients the cause is drug-induced toxicity. The median time for progression from Child-Pugh A to ChildPugh B or C was 2.9 months (95\% CI, 1.87-4.8 months). Nevertheless, median sorafenib concentrations in patients progressing to Child-Pugh B or C were similar to those in patients with Child-Pugh A, suggesting that sorafenib exposure was not associated with Child-Pugh status. A 


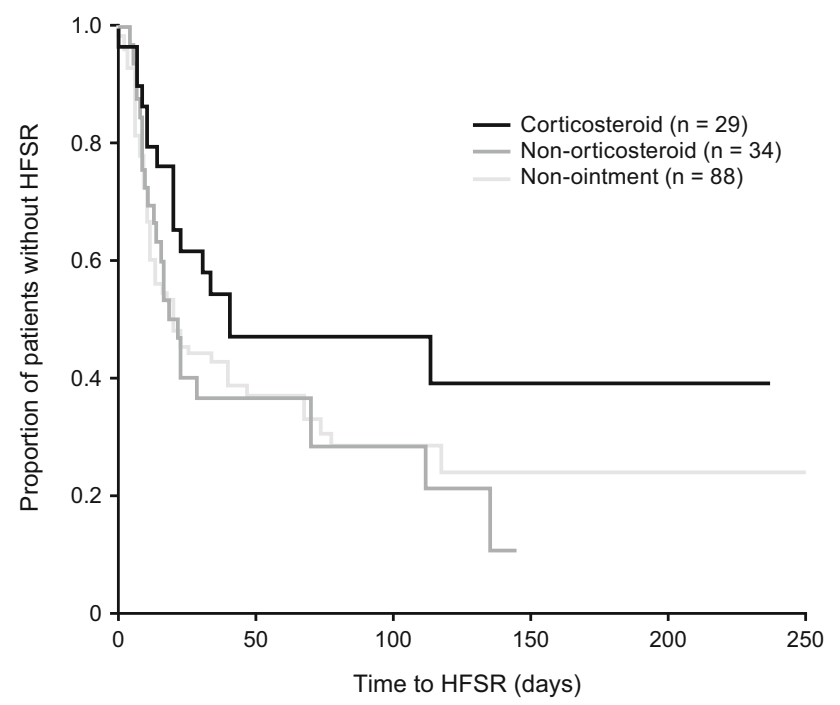

Fig. 4 Kaplan-Meier analysis of time to HFSR in sorafenib-treated HCC patients randomized to corticosteroid $(n=29)$ and noncorticosteroid $(n=34)$ ointments and in nonointment-treated patients from the HATT trial registered before the beginning of the HFSR prevention substudy $(n=88)$. HFSR hand-foot skin reaction, $H C C$ hepatocellular carcinoma, HATT Hepatocellular Carcinoma-Advanced Stage-Sorafenib Trial in Taiwanese patients

small number of patients $(n=12)$ had Child-Pugh $\mathrm{C}$ scores at the end of treatment. Although patient-level data on causes for change from Child-Pugh A to Child-Pugh C score were not collected, most patients who discontinued sorafenib therapy had disease progression. Disease progression may be a key contributor to the change from Child-Pugh A to Child-Pugh $\mathrm{C}$, although a separate prospective study is needed to determine predictors of change in Child-Pugh score in patients on sorafenib treatment. Of note, consistent progression over time in Child-Pugh scores (i.e., from A to B, B to C, and A to C) was not observed in all patients. Instead, in some patients, Child-Pugh scores varied within patients over time, with some patients showing improvements after initial worsening and others demonstrating progressive deterioration.

Application of a population pharmacokinetic model suggested that sorafenib concentrations tended to decrease over time. Concentrations of albumin and bilirubin were positively correlated with sorafenib concentrations, although only bilirubin concentration showed a time dependence, possibly due to sorafenib inhibiting metabolism of bilirubin by UGT1A1 [11]. The final pharmacokinetic model predicted decreases in sorafenib exposure over 6 and 12 months of 13.1 and $33.8 \%$, respectively. This change in concentration was not associated with reductions in liver function, as shown by Child-Pugh score, nor was it due to reductions or interruptions in sorafenib dosing. The mechanisms underlying these reductions in sorafenib exposure, and the clinical impact of these reductions, have not yet been determined. Nonlinear mixed effects modeling using NONMEM is based on datasets containing the actual doses taken and, should they occur, naturally adjusts the model predictions for dosing changes. Because the model predictions insufficiently accounted for reductions in observed concentrations, the changes are therefore caused by factors other than dosing reductions/interruptions.

\section{Hand-foot skin reaction}

HFSR is a frequent AE of tyrosine kinase inhibitors, including sorafenib, that target the vascular endothelial growth factor signaling pathway $[5,6,12-16]$. Topical steroids have been reported effective in the treatment of sorafenib-induced HFSR [17-19]. Analysis of incidence, time to HFSR, and HFSR score in a subset of patients in this study randomized to a corticosteroid or a noncorticosteroid cream found that those randomized to the corticosteroid cream tended to have a longer time to onset of HFSR and a better HFSR score than patients randomized to the noncorticosteroid cream, although most differences were not statistically significant. Interestingly, although the initial cohort of patients not prescribed ointment before the randomized HFSR prevention substudy had similar HFSR incidence, time to HFSR, and HFSR scores as patients randomized to noncorticosteroid cream, the incidence of grade 3 HFSR was noticeably lower in the noncorticosteroid cream group than in the nonointment group (i.e., the initial 88 nonrandomized patients), potentially supporting the value of any type of ointment for HFSR prophylaxis. A recent trial involving 871 Chinese patients treated for HCC found that prophylactic administration of a urea-based cream significantly reduced the 12-week incidence of any grade HFSR $(56.0 \%$ vs. $73.6 \%$; OR, 0.457 ; $95 \% \mathrm{CI}$, $0.344-0.608 ; p<0.0001)$ and of grade $\geq 2$ HFSR $(20.7 \%$ vs. $29.2 \% \%$; OR, $0.635 ;$; $95 \%$ CI, $0.466-0.866 ; p=0.004$ ) compared with best-supportive care alone [19]. Moreover, the median time to first occurrence of HFSR was significantly longer in the urea-based cream than in the control group (84 vs. 34 days; hazard ratio, $0.658 ; 95 \%$ CI, $0.541-0.799 ; p<0.0001)$. Taken together, these findings suggest that prophylactic treatment with a urea-based or corticosteroid-containing cream may benefit patients being treated with sorafenib for HCC.

Although, in the main study, AEs such as HFSR, ascites, and diarrhea were frequent, all were manageable and rarely resulted in discontinuation from treatment. There were no new or unexpected safety findings.

In conclusion, the outcome of this study confirms the results of the previous Sorafenib AP study. The pharmacokinetic model predicted decreases in sorafenib exposure, but the clinical significance of the observed $33.8 \%$ decrease in exposure after 1 year remains unclear, especially given the shorter median and mean treatment duration in this 
patient population. Sorafenib remains the standard of care for Asian patients with advanced/metastatic HCC.

Acknowledgements This study was supported by Bayer HealthCare Pharmaceuticals and Onyx Pharmaceuticals, an Amgen subsidiary. Editorial assistance was provided to the authors by BelMed Professional Resources, with funding by Bayer HealthCare Pharmaceuticals.

\section{Compliance with ethical standards}

Conflict of interest Shi-Ming Lin, Sheng-Nan Lu, Ping-Tsung Chen, Long-Bin Jeng, Shinn-Cherng Chen, Chi-Tan Hu, Sien-Sing Yang, and Klaas Prins declare that they have no conflicts of interest. MarieAude Le Berre, Xuan Liu, Carol Peña, and Gerold Meinhardt are employees of Bayer HealthCare Pharmaceuticals. David Y. Mitchell is a paid consultant for Bayer Healthcare Pharmaceuticals. Joachim Grevel is an employee of BAST Inc Limited and paid consultant for Bayer Healthcare Pharmaceuticals.

Ethical approval All procedures were in accordance with the ethical standards of the responsible committee on human experimentation (institutional and national) and with the Helsinki Declaration of 1975, as revised in 2008. The trial protocol was approved by the institutional review boards of all seven institutions. The trial has been registered at clinicaltrials.gov as NCT01098760.

Informed consent Informed consent was obtained from all patients for being included in the study.

Open Access This article is distributed under the terms of the Creative Commons Attribution 4.0 International License (http://crea tivecommons.org/licenses/by/4.0/), which permits unrestricted use, distribution, and reproduction in any medium, provided you give appropriate credit to the original author(s) and the source, provide a link to the Creative Commons license, and indicate if changes were made.

\section{References}

1. The GLOBOCAN project. Lyon, France: International Agency for Research on Cancer, 2010. Available at: http://globocan.iarc. fr. Accessed 4 Nov 2015

2. Parkin DM, Bray F, Ferlay J, Pisani P. Global cancer statistics, 2002. CA Cancer J Clin. 2005;55(2):74-108

3. Hussain SA, Ferry DR, El-Gazzaz G, et al. Hepatocellular carcinoma. Ann Oncol. 2001;12(2):161-172

4. Katherine A, Hashem B. Global incidence of hepatocellular carcinoma. In: Bruix J, editor. Hepatocellular carcinoma. Barcelona: Permanyer; 2004
5. Cheng AL, Kang YK, Chen Z, et al. Efficacy and safety of sorafenib in patients in the Asia-Pacific region with advanced hepatocellular carcinoma: a phase III randomised, double-blind, placebo-controlled trial. Lancet Oncol. 2009;10(1):25-34

6. Llovet JM, Burroughs A, Bruix J. Hepatocellular carcinoma. Lancet. 2003;362(9399):1907-1917

7. Prins K, Ploeger BA. Population pharmacokinetic analysis of sorafenib plasma concentrations in Phase II through Phase IV monotherapy studies. Bayer Internal Report R-8977; September 2013

8. Prins NH, Grevel J, Mitchell D, et al. Population pharmacokinetics of sorafenib: a meta-analysis of patients with hepatocellular carcinoma, renal cell carcinoma, and differentiated thyroid carcinoma [Abstract]. J Pharmacokinet Pharmacodyn. 2014;41(Suppl):s23

9. Cheng AL, Kang YK, Lin DY, et al. Sunitinib versus sorafenib in advanced hepatocellular cancer: results of a randomized phase III trial. J Clin Oncol. 2013;31(32):4067-4075

10. Johnson PJ, Qin S, Park JW, et al. Brivanib versus sorafenib as first-line therapy in patients with unresectable, advanced hepatocellular carcinoma: results from the randomized phase III BRISK-FL study. J Clin Oncol. 2013;31(28):3517-3524

11. Peer CJ, Sissung TM, Kim A, et al. Sorafenib is an inhibitor of UGT1A1 but is metabolized by UGT1A9: implications of genetic variants on pharmacokinetics and hyperbilirubinemia. Clin Cancer Res. 2012;18(7):2099-2107

12. Beldner M, Jacobson M, Burges GE, et al. Localized palmarplantar epidermal hyperplasia: a previously undefined dermatologic toxicity to sorafenib. Oncologist. 2007;12(10):1178-1182

13. Lacouture ME, Reilly LM, Gerami P, Guitart J. Hand foot skin reaction in cancer patients treated with the multikinase inhibitors sorafenib and sunitinib. Ann Oncol. 2008;19(11):1955-1961

14. Robert C, Soria JC, Spatz A, et al. Cutaneous side-effects of kinase inhibitors and blocking antibodies. Lancet Oncol. 2005;6(7):491-500

15. Yang $\mathrm{CH}$, Lin WC, Chuang $\mathrm{CK}$, et al. Hand-foot skin reaction in patients treated with sorafenib: a clinicopathological study of cutaneous manifestations due to multitargeted kinase inhibitor therapy. Br J Dermatol. 2008;158(3):592-596

16. Zhang L, Zhou Q, Ma L, Wu Z, Wang Y. Meta-analysis of dermatological toxicities associated with sorafenib. Clin Exp Dermatol. 2011;36(4):344-350

17. Cuesta L, Betlloch I, Toledo F, Latorre N, Monteagudo A. Severe sorafenib-induced hand-foot skin reaction. Dermatol Online J. 2011;17(5):14

18. Manchen E, Robert C, Porta C. Management of tyrosine kinase inhibitor-induced hand-foot skin reaction: viewpoints from the medical oncologist, dermatologist, and oncology nurse. J Support Oncol. 2011;9(1):13-23

19. Ren Z, Zhu K, Kang H, et al. Randomized controlled trial of the prophylactic effect of urea-based cream on sorafenib-associated hand-foot skin reactions in patients with advanced hepatocellular carcinoma. J Clin Oncol. 2015;33(8):894-900 